\title{
Impacto de la autopresentación perfeccionista en la salud infantil y adolescente: Una revisión sistemática
}

\author{
Sonia Chemisquy y Gisela P. Helguera \\ Universidad de la Cuenca del Plata, Posadas, Argentina
}

Impact of perfectionistic self-presentation on children and adolescents' health: A systematic review

\begin{abstract}
Perfectionistic self-presentation is an interpersonal style in which the person attempts to actively show perfection and avoids displaying and disclosing any imperfections. The aim of this study was to review the scientific literature in order to determine the impact of perfectionistic self-presentation on children and adolescents' health. Scientific evidence was found on the presence of perfectionistic self-presentation from childhood as a related yet differential construct from perfectionism, that negatively impacts on children and adolescents' mental health through increased interpersonal conflicts, psychological vulnerability and risk of developing anxiety, eating disorders and depression.
\end{abstract}

Keywords: Perfectionistic self-presentation; children; adolescent; psychological vulnerability.

Resumen: La autopresentación perfeccionista es un estilo interpersonal caracterizado por los intentos de mostrar activamente perfección, de ocultar los errores y no hablar de ellos. El objetivo de este trabajo fue revisar la literatura científica para determinar el impacto de la autopresentación perfeccionista en la salud de niños y adolescentes. Los artículos científicos revisados evidencian la presencia de la autopresentación perfeccionista desde la infancia como un constructo diferenciado del perfeccionismo que incide negativamente en la salud mental de niños y adolescentes aumentando los conflictos interpersonales, la vulnerabilidad psicológica y el riesgo para desarrollar ansiedad, trastornos de la conducta alimentaria y depresión.

Palabras clave: Autopresentación perfeccionista; niños; adolescentes; vulnerabilidad psicológica.

\section{Introducción}

El perfeccionismo desadaptativo se define como la tendencia a la autoimposición de metas irracionalmente elevadas, donde predomina una autoevaluación excesivamente crítica, y la medición del valor propio se configura en función de los logros y los fracasos (Hewitt y Flett, 1991). La investigación indica que, al menos en parte, su origen puede encontrarse en el ambiente familiar (Maloney, Egan, Kane, y Rees, 2014) donde se establecen pautas de apego inseguro (Chen, Hewitt, y Flett, 2015) en relación a las cuales se conforma un esquema desadaptativo temprano de imperfección (Caputto, Cor-

Recibido: 18 de octubre 2017; aceptado: 17 de marzo 2018

Correspondencia: Sonia Chemisquy, Universidad de la Cuenca del Plata, Barrufaldi 2364, 3300 Posadas, Argentina.

Correo-e: chemisquysonia_pos@ucp.edu.ar dero, Keegan, y Arana, 2015). Propias de este esquema son las ideas autoderrotistas acerca de uno mismo como alguien defectuoso, imperfecto y fracasado.

Los esquemas desadaptativos tempranos representan un marco desde el cual las personas dan sentido a la experiencia y suelen estar en la base de los trastornos mentales. Por lo general toman forma a partir de la insatisfacción o, al contrario, la satisfacción de necesidades básicas del desarrollo (Young, Klosko, y Weishaar, 2003). En los perfeccionistas desadaptativos, junto al esquema de imperfección, se han encontrado otros pertenecientes al dominio de la desconexión/rechazo (Jahromi, Naziri, y Barzegar, 2012), que explican las dificultades experimentadas en el vínculo de apego de estas personas.

Los perfeccionistas desadaptativos no solo experimentan problemas relacionados con los logros y/o a nivel cognitivo. Además, suelen tener una serie de dificul- 
tades en el ámbito interpersonal que se relacionan tanto con la necesidad de aceptación como con la escasa habilidad para auto-reforzar los propios logros, lo que vuelve a estas personas más dependientes del refuerzo externo (Wu y Wei, 2008). Sin embargo, la búsqueda de refuerzo y de aceptación se ve obstaculizada por el temor a ser evaluados de manera negativa por los demás y por otros índices de sensibilidad interpersonal (p.ej. actitudes dependientes, timidez, percepción de que los demás son distantes) que llevan a los perfeccionistas a interpretar de manera negativa las relaciones sociales (Sherry, Mackinnon, y Gautreau, 2016). De esta forma, cuando los perfeccionistas desadaptativos se enfrentan al otro se activan las creencias de que son personas defectuosas junto a la necesidad de ser aceptados. Por esta razón, se ven empujados a exagerar sus rasgos de perfección y a ocultar sus errores como una manera de sobrellevar el temor a la evaluación social negativa y en vistas de que consideran que sólo serán aceptados y amados en función de sus logros y/o fracasos.

En términos generales, cuando las personas se encuentran ante la inminente evaluación de los demás se pueden activar una serie de comportamientos de autopresentación cuya finalidad es manipular la información personal que se va a compartir con los otros. Si bien la manera en que una persona se autopresenta está influenciada por el contexto, los individuos suelen tener un estilo autopresentacional que tiende a ser estable en el tiempo y que se fundamenta en la motivación que subyace a la forma de presentarse ante los otros (Baumeister y Hutton, 1987).

Algunos perfeccionistas desadaptativos utilizan la autopresentación perfeccionista en sus relaciones interpersonales como un estilo interaccional permanente con el que manejan la imagen que muestran a los demás para lograr mostrarse como ideales y perfectos (Chen et al., 2015). Mientras que el perfeccionista se afana por alcanzar sus estándares de perfección y ser perfecto, quien se presenta a sí mismo de manera perfeccionista se esfuerza para que los demás perciban la imagen de su aparente excelencia (Hewitt et al., 2003).

La autopresentación perfeccionista contiene dos componentes motivacionales: mostrar la propia perfección y ocultar las imperfecciones. A partir de estas dos motivaciones se configuran tres facetas que hacen de este estilo autopresentacional un constructo multidimensional (Hewitt et al., 2003): la autopromoción de la propia perfección, no mostrar las imperfecciones y no revelar las imperfecciones. La autopromoción de la propia perfección es una faceta representada por los esfuerzos activos que realiza la persona por exhibir sus logros y por exponerse ante los demás como alguien perfecto. En esta vertiente se integra la motivación por mostrar a los demás la imagen de uno mismo como alguien perfecto, objetivo que se logra ostentando las conquistas personales, exagerando los logros y, en definitiva, esforzándose por lucir y comportarse perfectamente frente a los demás.

Las otras dos facetas, no mostrar las imperfecciones y no revelar las imperfecciones, tienen su fundamento en la preocupación por ocultar las equivocaciones y representan el aspecto más pasivo de este constructo. Para no mostrar sus imperfecciones o fallos, las personas que se presentan a sí mismas como perfectas evitan las situaciones en las que vaticinan probabilidades de fracasar (p.ej., participar en un deporte para el que no tienen suficiente habilidad), se aislan para no ser vistas (p.ej. elegirán una academia de idiomas en un barrio alejado, comprobando que a este lugar no asisten sus allegados), $\mathrm{u}$ ocultan o destruyen las evidencias de sus fracasos (p.ej., esconderán el boletín de calificaciones o notas). No revelar las imperfecciones o errores, por su parte, lo logran ocultando aquellas cosas percibidas como imperfecciones: las notas bajas, el partido de fútbol perdido e incluso los pensamientos o emociones negativas, que en sí mismos pueden ser experimentados como inaceptables (James, Verplanken y Rimes, 2015). Estas dos facetas llevan a cabo el disimulo de los fracasos de maneras distintas: mientras que en la primera prevalecen los aspectos comportamentales (p.ej., dejar de hacer cosas para no evidenciar los errores), en la segunda prima el componente verbal (p.ej., no hablar de las equivocaciones).

Desde los primeros estudios sobre el tema, los investigadores han encontrado que la autopresentación perfeccionista se asocia al perfeccionismo, particularmente al auto-orientado y al socialmente prescrito (Hewitt et al., 2003). Además, se asocia a algunos factores de vulnerabilidad psicológica tales como la baja autoestima, los síntomas de ansiedad y depresión, la preocupación por las evaluaciones sociales sobre la apariencia y la preocupación por las evaluaciones negativas (Hewitt, Flett, y Ediger, 1995; Hewitt et al., 2003). Por último, se ha encontrado que la autopresentación perfeccionista está en la base de algunos trastornos mentales tales como los de la conducta alimentaria (Hewitt et al., 1995) y los de la personalidad (Sherry, Hewitt, Flett, Lee-Baggley, y Hall, 2007).

La mayoría de las investigaciones sobre esta temática estudian su comportamiento en población universitaria o adulta, siendo muy escasa la evidencia empírica con muestras infantiles o adolescentes. En nuestro contexto hispanohablante se percibe aún más la carencia de estudios sobre la temática. 
Esta escasez de conocimiento refleja el desafío que representa la detección de este tipo de problemas de salud mental (Flett y Hewitt, 2013). Así, en el contexto de la práctica clínica es habitual encontrarse con niños que han desarrollado alguna manifestación sintomática que sorprende a sus familiares a y su ambiente más cercano ya que hasta ese momento no habían expresado ningún indicio de su malestar, al contrario, parecían estar totalmente adaptados y ajustados a sus circunstancias vitales. Al igual que ocurre en población universitaria y adulta, sería esperable que los niños y adolescentes que se presenten a sí mismos de manera perfeccionista experimenten conflictos interpersonales, vean aumentada su vulnerabilidad psicológica y estén en riesgo para desarrollar trastornos de la salud mental.

Teniendo en cuenta las lagunas en la investigación señaladas anteriormente, el objetivo de este trabajo es realizar una revisión sistemática de las principales publicaciones científicas encontradas hasta el momento sobre la temática, intentando dar respuesta a una serie de interrogantes: (a) ¿cómo se relacionan el perfeccionismo y la autopresentación perfeccionista?, (b) ¿cuáles son los correlatos psicológicos y sociales de la autopresentación perfeccionista en la infancia y la adolescencia? y (c) ¿con qué trastornos psicológicos se asocia a autopresentación perfeccionista en población infanto-juvenil?

\section{Método}

Durante el mes de junio de 2017 se realizó una búsqueda en las bases de datos Pubmed, ScienceDirect, Springerlink, Scielo y Redalyc utilizando el siguiente conjunto de términos: perfectionistic AND self-presentation OR presentation AND perfection* OR perfeccionis* AND presentación OR perfeccionis* AND autopresentación. Los términos seleccionados fueron lo suficientemente amplios para poder recolectar la mayor cantidad de información posible y no pasar por alto estudios importantes. En dos casos (Springerlink y Redalyc) se aplicaron filtros según la disciplina para obtener artículos pertenecientes a Psicología y Medicina y Salud. La misma búsqueda se realizó en el mes de marzo de 2018 con objetivo de actualizar los resultados obtenidos.

Los artículos científicos obtenidos fueron seleccionados si cumplían los siguientes criterios de inclusión: (a) los estudios debían evaluar la autopresentación perfeccionista con algún instrumento psicométrico, (b) los artículos debían describir estudios empíricos, (c) la edad de la muestra utilizada por los estudios debía comprender, al menos en parte, el grupo etario de 8 a 17 años, y (d) el idioma de las publicaciones debía ser inglés o español.

Cada uno de los estudios fue analizado para obtener información acerca de sus autores y año de publicación, el país de origen y el idioma en que fueron publicados, el tamaño de la muestra utilizada, las edades de los participantes, el instrumento de evaluación utilizado para medir el constructo autopresentación perfeccionista, las hipótesis, los objetivos y los principales resultados encontrados.

\section{Resultados}

Se encontraron un total de 14 artículos que cumplían los criterios de inclusión, de los cuales 13 fueron publicados en idioma inglés y solo uno en español. En la Figura 1 se detalla el proceso de selección de los estudios. El resumen de los datos de cada uno de los artículos y de sus resultados principales se encuentra en la Tabla 1.

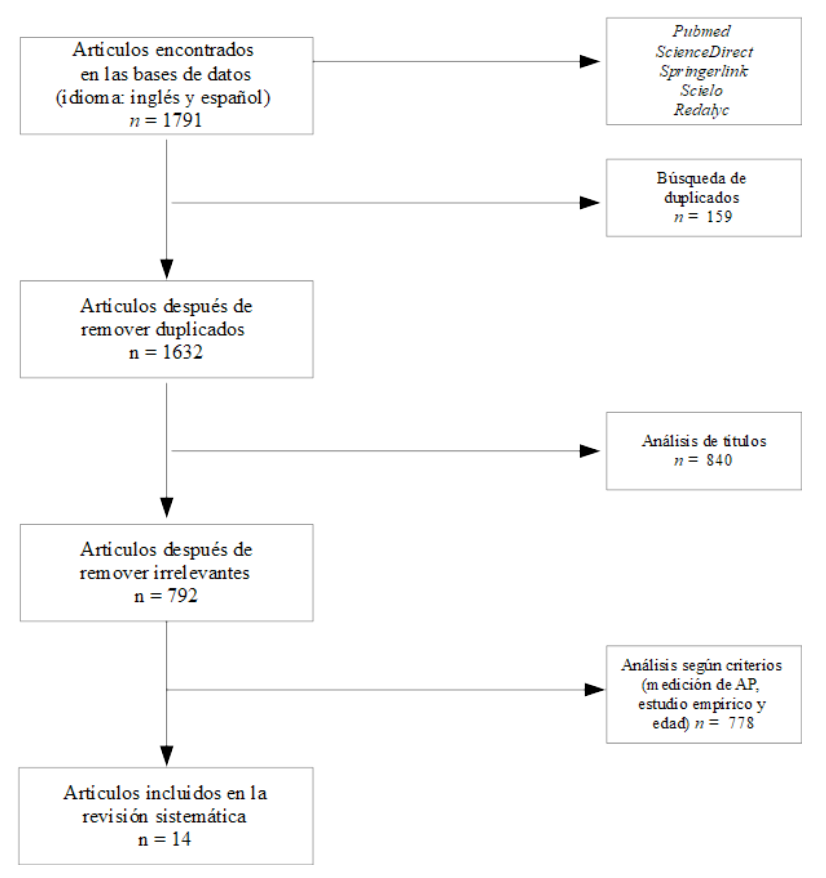

Figura 1. Diagrama de flujo del proceso de selección de artículos.

Como puede observarse, el criterio de selección basado en la edad tuvo que ser modificado en función de los resultados hallados, ya que muchos de los artículos utilizaron muestras de edades que no se correspondían exactamente con el rango seleccionado. Por ejemplo, Bardone-Cone, Sturm, Lawson, Robinson y Smith (2010) seleccionan mujeres de 16 años en adelante, tomando sólo un sector del rango establecido como criterio, pero debido a la pertinencia del análisis en la adolescencia fue incluido en este estudio de revisión. Esto mismo ocurrió con los estudios de Boone (2013), Chen et al. (2012), Castro et al. (2014), Ferreira, Trindade, y Ornelas (2015), Hewitt et al. (2011), Roxborough et al. (2012), y Vuyk (2015). 


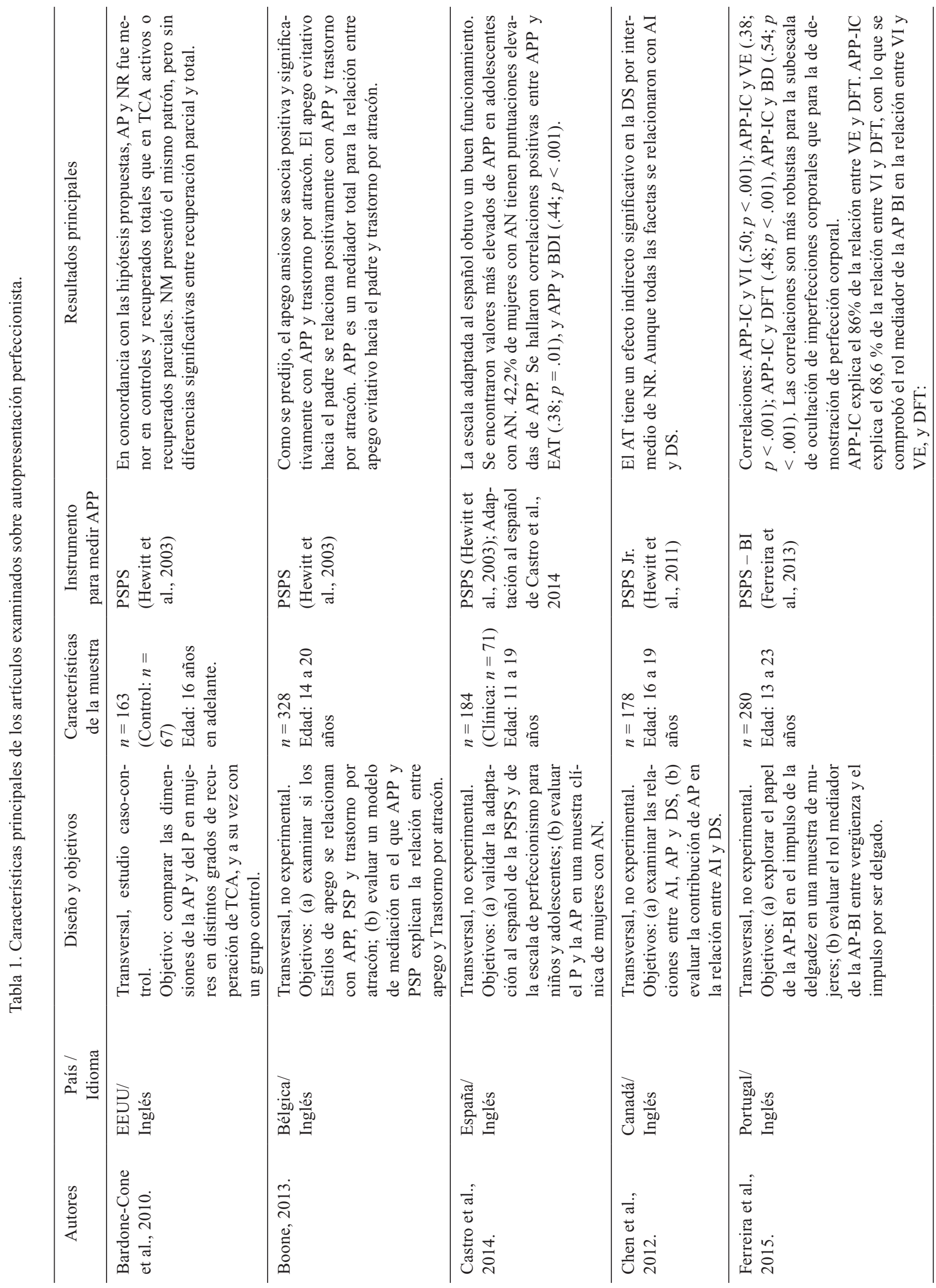




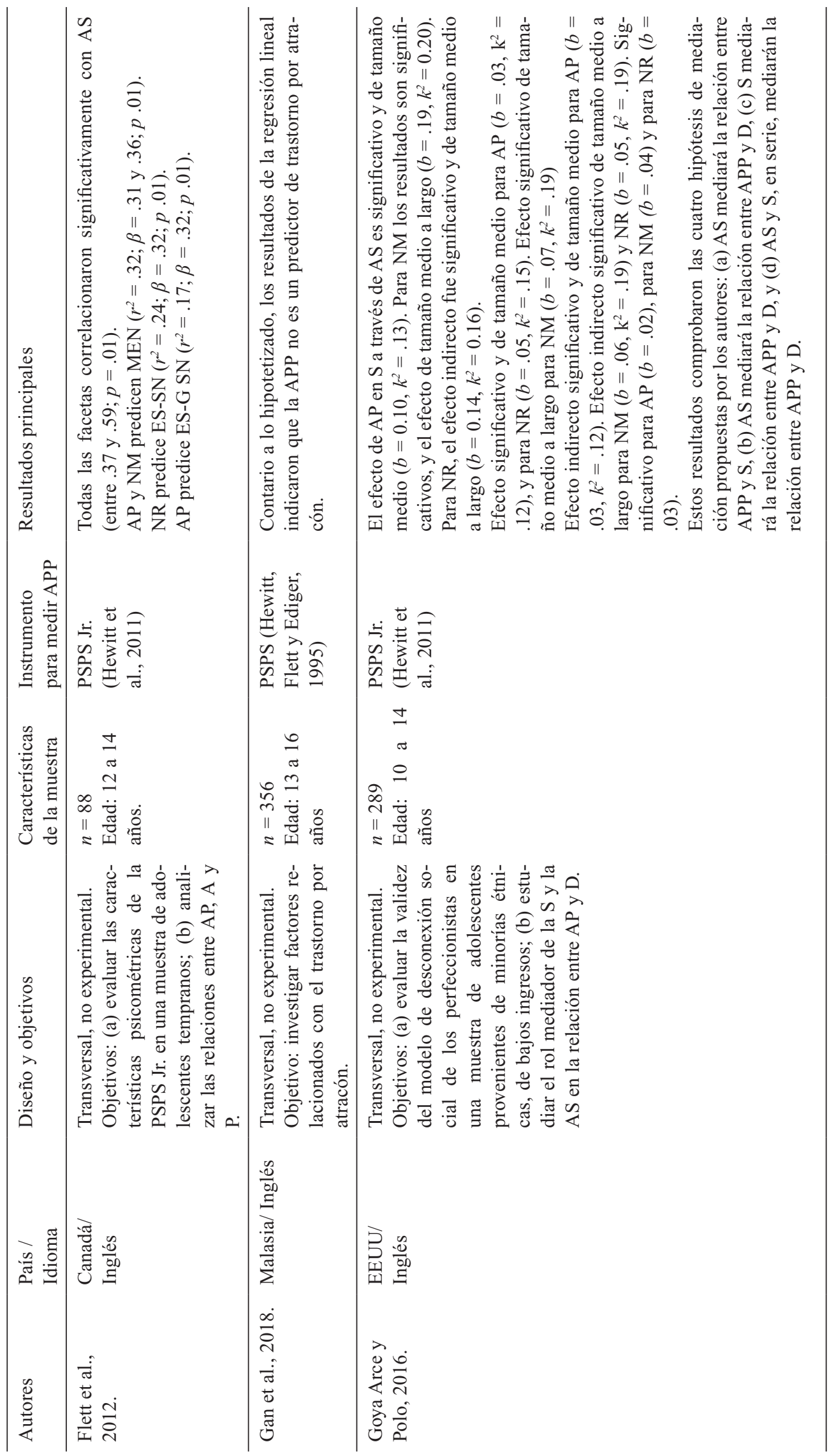




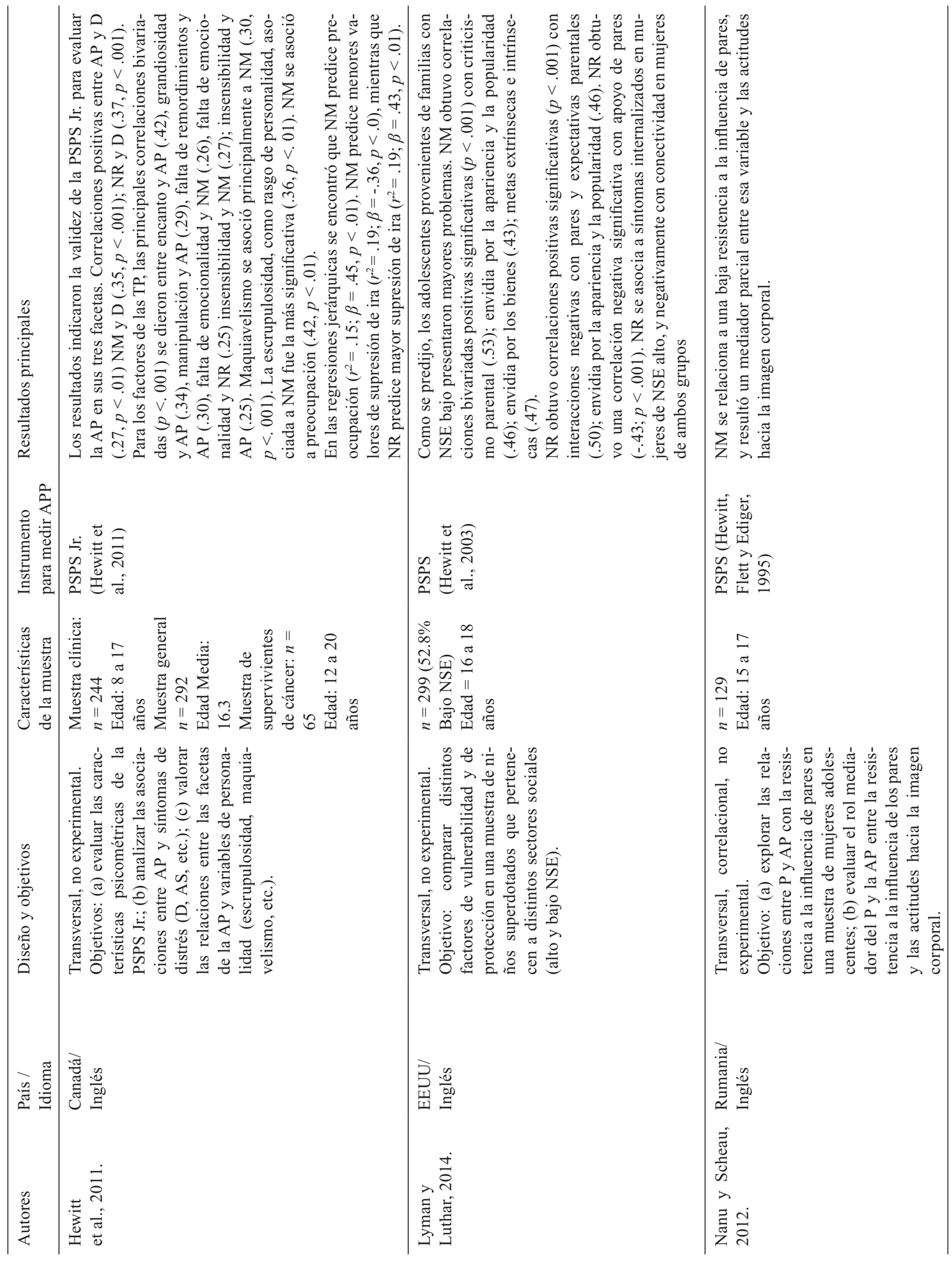




\begin{tabular}{|c|c|c|c|c|}
\hline 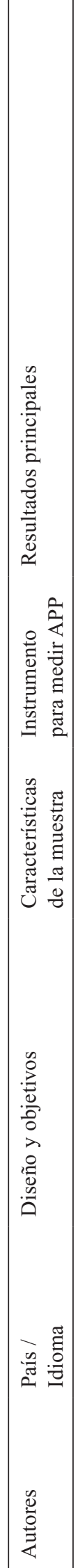 & 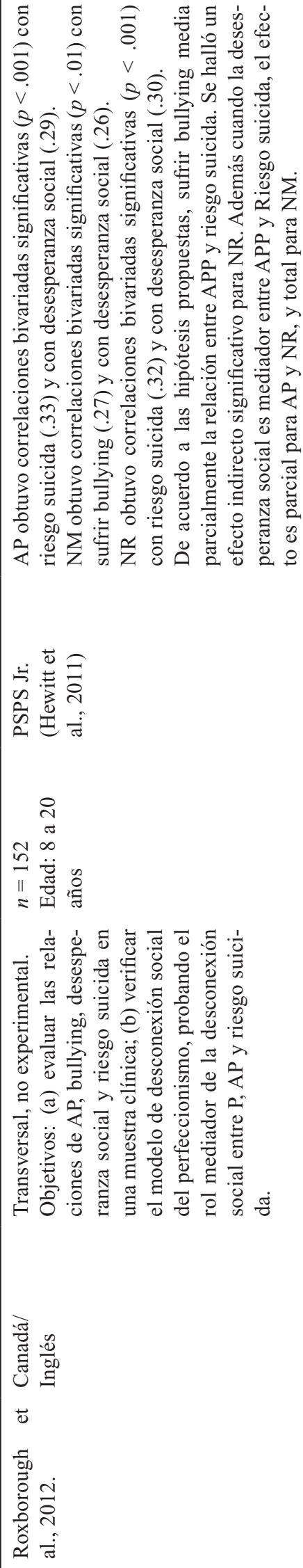 & 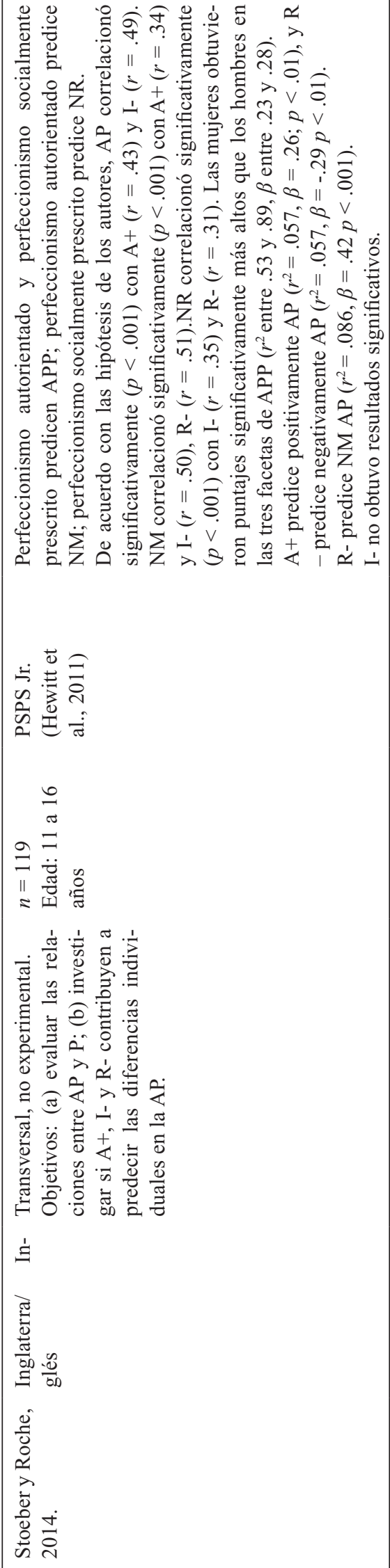 & 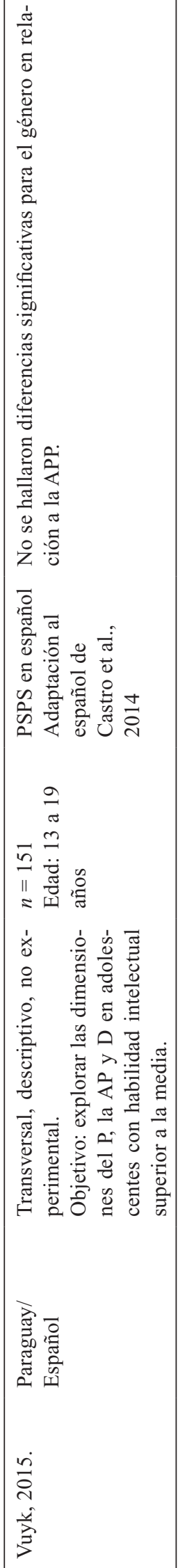 & 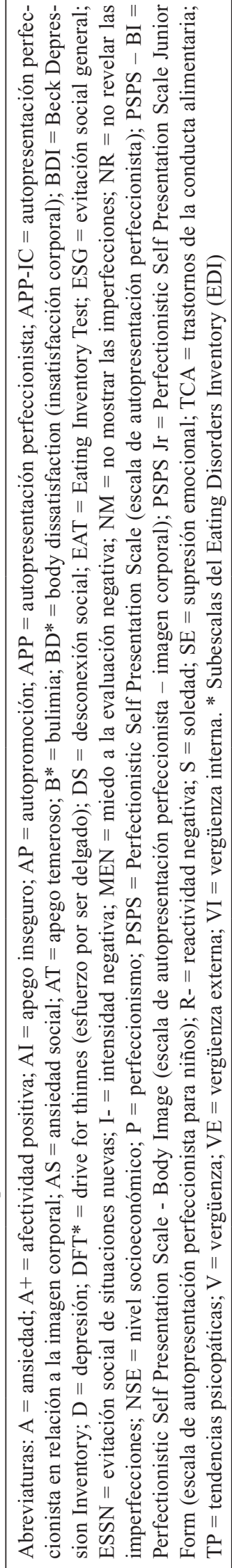 \\
\hline
\end{tabular}


En términos generales, la revisión de la literatura científica mostró que la autopresentación perfeccionista a se relacionaba con malestar experimentado en diversas áreas pero principalmente dos: 1) el aumento de la vulnerabilidad psicológica en forma de disposiciones de personalidad o de problemas interpersonales y 2) el desarrollo de trastornos psicológicos como la ansiedad social, los trastornos de la conducta alimentaria, o la depresión.

\section{Autopresentación perfeccionista y perfeccionismo}

En los artículos científicos revisados, las tres facetas de la autopresentación perfeccionista obtuvieron correlaciones diferenciales con los distintos tipos de perfeccionismo (Boone, 2013; Flett Coulter y Hewitt, 2012; Hewitt et al., 2011; Roxborough et al., 2012; Stoeber y Roche, 2014).

Para el perfeccionismo auto-orientado (i.e., la autoimposición de estándares elevados), las correlaciones con la autopromoción de la propia perfección resultaron significativas y robustas ( $r$ de Pearson entre .46 y .61), al igual que con la faceta de no mostrar las imperfecciones ( $r$ de Pearson entre .38 y .58). Por su parte, para la dimensión de no revelar las imperfecciones se obtuvieron correlaciones bivariadas de Pearson entre .23 y .30 que indican una correlación media (Flett, Coulter y Hewitt, 2012; Hewitt et al., 2011; Nanu y Scheau, 2013; Roxborough et al., 2012; Stoeber y Roche, 2014).

El perfeccionismo socialmente prescrito (i.e., la percepción de que los otros significativos demandan perfección) correlacionó de manera significativa y robusta con la autopromoción de la propia perfección ( $r$ de Pearson entre .38 y .52), mientras que las correlaciones bivariadas de Pearson entre esta dimensión del perfeccionismo como rasgo y no mostrar imperfecciones oscilaron entre .10 y .42 , y para la faceta de no revelar las imperfecciones los índices de correlación $r$ de Pearson se ubicaron entre .34 y .39 (Flett, Coulter y Hewitt, 2012; Hewitt et al., 2011; Nanu y Scheau, 2013; Roxborough et al., 2012; Stoeber y Roche, 2014). En el estudio de Stoeber y Roche (2014) se describe el patrón de correlaciones entre las tres facetas de la autopresentación perfeccionista y el perfeccionismo. Los jóvenes que tienden a la autopromoción de la propia perfección suelen tener elevados niveles de perfeccionismo auto-orientado y perfeccionismo socialmente prescrito; aquellos que prefieren no mostrar las imperfecciones, suelen tener sólo niveles altos de perfeccionismo auto-orientado $\mathrm{y}$, por último, los que no revelan las imperfecciones suelen presentar mayores niveles de perfeccionismo socialmente prescrito.
Correlatos psicológicos y sociales de la autopresentación perfeccionista

Si bien es habitual la asociación entre autopresentación perfeccionista y neuroticismo en los adultos, esta relación no está establecida en etapas previas del desarrollo (Sherry et al., 2007). En la población estudiada Hewitt y sus colaboradores (2011) han encontrado que la escrupulosidad es el rasgo de personalidad con relaciones más robustas, en particular para aquellos que autopromocionan sus propias perfecciones y para los que no muestran sus imperfecciones. En este mismo estudio, la faceta de no revelar las imperfecciones no ha obtenido correlaciones significativas con ninguno de los cinco grandes rasgos de la personalidad en población infantil y adolescente.

La autopresentación perfeccionista se asocia con las actitudes disfuncionales, entendidas como un conjunto de esquemas cognitivos de tipo depresógeno con el que se interpreta la información proveniente del medio (D'Alessandro y Burton, 2006). A partir de esta asociación, se explican los pensamientos condicionales (i.e., si... entonces...) y la autovaloración contingente (i.e., si fallo, entonces soy un fracaso) característicos de la autopresentación perfeccionista (Flett et al., 2012). El procesamiento de información mediado por estas actitudes disfuncionales está sesgado y da como resultado las creencias propias de la tríada cognitiva: una visión negativa acerca de uno mismo, del mundo y del futuro (Beck, Rush, Shaw y Emery, 2008), por lo que suponen el aumento de la vulnerabilidad y el malestar psicológico.

Se pueden describir perfiles diferenciales en relación a la predominancia de cada una de las facetas. Los niños y adolescentes que tienden a no revelar sus imperfecciones suelen expresar escasamente sus emociones y suprimen la ira, mientras que los que promocionan sus propias perfecciones suelen tener un estilo narcisista, caracterizado por la grandiosidad y el encanto, por lo que utilizan la promoción de sus rasgos ideales para lograr la admiración y el respeto de los demás. Por su parte, los que prefieren no mostrar sus imperfecciones son quienes experimentan mayores niveles de malestar psicológico, en la forma de síntomas depresivos, ansiedad, etc. (Hewitt et al., 2011).

Aunque algunos autores (Hewitt et al., 2011) proponen que los niños y adolescentes que se autopresentan de manera perfeccionista carecen de emocionalidad, o al menos aparentan desapego emocional, los hallazgos de Stoeber y Roche (2014) describen la presencia de patrones de emocionalidad para las distintas facetas. Así, aquellos que prefieren promocionar su propia perfección tienden a experimentar más afectividad positiva y a tener 
baja reactividad a los sucesos negativos y a la inversa, los niños y adolescentes que no muestran las imperfecciones son más propensos a reaccionar a los eventos negativos, al tiempo que experimentan escasa afectividad positiva. Según estos últimos autores, estos datos permiten predecir la faceta que manifestarán los niños y adolescentes de forma que los que experimentan altos niveles de afectividad positiva optarán por mostrar sus propias perfecciones, mientras que aquellos con niveles elevados de reactividad a las experiencias negativas y escasas emociones positivas preferirán no mostrar las imperfecciones.

Además, estos jóvenes autopresentadores suelen sentir envidia respecto de sus pares. Puntualmente, Lyman y Luthar (2014) hallaron que los adolescentes que se autopresentan de manera perfeccionista envidian el aspecto físico, la popularidad y la riqueza de sus pares. Según estos investigadores la envidia puede motivar la urgencia de aparentar perfección, ya que al realizar una comparación social encuentran en los otros atributos deseados para sí mismos (Smith y Kim, 2007). Esta comparación desfavorable puede aumentar los sentimientos de inferioridad ya experimentados por estos niños y adolescentes, y activar así los comportamientos de autopresentación perfeccionista.

Otro elemento a tener en cuenta es el establecimiento de metas externamente orientadas, para las cuales la motivación emana de la búsqueda de recompensas y/o de la evitación de resultados negativos, ambos provenientes de agentes externos. En general, se acepta que las personas que establecen metas externas son más propensas a experimentar depresión, ansiedad y sentimientos de baja autoestima (Deci y Ryan, 2012). El establecimiento de metas externas es un elemento de considerable interés ya que, tanto para los perfeccionistas como para aquellos que se presentan de manera perfeccionista, alcanzar las metas es un objetivo de gran importancia. Cuando la motivación para alcanzarlas es externa, las personas quedan a merced de los demás, que tienen el poder de definir sus logros y fracasos. Asociado a esto se encontró que las mujeres que promocionan sus perfecciones y que no muestran sus errores son menos resistentes a las influencias de sus pares, por lo que pueden imponerse la tarea de conseguir y/o mostrar ciertas características socialmente deseadas (imagen, peso, etcétera) con el fin de lograr conformidad social (Nanu y Scheau, 2013).

Se puede pensar que la tendencia al establecimiento de metas externas puede llevar a que estos niños y adolescentes aumenten el afán por mostrarse perfectos, en particular si se tiene en cuenta que, para los perfeccionistas, la autovaloración suele ser contingente a los logros y fracasos. Los autopresentadores exaltarán las metas alcanzadas y ocultarán las equivocaciones como estrategia para incrementar el autovalor y conseguir aceptación de los demás.

Chen y sus colaboradores (2012) encontraron que la autopresentación perfeccionista se asocia a un estilo de apego inseguro, ya sea temeroso, preocupado o ansioso. Esos hallazgos llevan a estos autores a concluir que los niños y adolescentes que se autopresentan de manera perfeccionista, están motivados a instrumentar este estilo interaccional para conseguir la aceptación y aprobación de los demás.

En el estudio de Boone (2013) se encontró una relación inversa entre apego seguro y autopresentación perfeccionista y además indicios de un rol diferencial del padre y de la madre en el establecimiento de un estilo de apego inseguro que lleva a la autopresentación perfeccionista. En la investigación de esta autora, el apego evitativo con el padre estuvo relacionado con la autopromoción de la propia perfección, mientras que el apego con la madre mostró más asociaciones con el perfeccionismo socialmente prescrito. Por lo tanto, la investigadora concluye que la autopresentación perfeccionista puede ser entendida como una estrategia para sobrellevar los sentimientos de malestar e imperfección asociados al vínculo distante con el padre.

Aunque los esfuerzos por aparentar perfección y ocultar los fracasos, propios de la autopresentación perfeccionista, parten de la necesidad de ser aprobados, resultan en un menoscabo de la vida interpersonal de niños y adolescentes: disminuyen el atractivo para los demás ya que pueden parecer fingidos al aparecer como siempre perfectos, aparentan ser elusivos por su tendencia a alejarse de las situaciones en las que se puedan evidenciar sus errores, y pueden parecer poco expresivos e insensibles porque no hablan de sus equivocaciones.

Estas características pueden hacer que los niños y adolescentes autopresentadores sean víctimas de bullying, o que sufran de otras modalidades de rechazo de parte de sus pares. En su fuero interno pueden percibir desconexión social subjetiva tanto en la forma de sentimientos de soledad, como de desesperanza respecto a la posibilidad de futuras relaciones sociales satisfactorias (Chen et al., 2012, Goya Arce y Polo, 2016; Lyman y Luthar, 2014, Roxborough et al., 2012).

Teniendo en cuenta que el apoyo social y las relaciones interpersonales pueden servir de un recurso útil al momento de afrontar situaciones de estrés y malestar, los problemas en el ámbito interpersonal pueden aumentar la vulnerabilidad psicológica. Así, siguiendo el modelo de desconexión social (Hewitt, Flett, Caelian y Sherry, 2006), el desarrollo de desenlaces negativos estaría supeditado a los problemas que experimentan en sus relaciones sociales. 


\section{Autopresentación perfeccionista y ansiedad}

Sobre la asociación entre autopresentación perfeccionista y ansiedad, los resultados de las investigaciones revisadas no indican un patrón claro que la explique. Hewitt y sus colegas (2011) encontraron que las facetas pasivas (no mostrar y no revelar imperfecciones) son las más relacionadas con la ansiedad, el estudio de Flett, Coulter y Hewitt (2012) halló que son la autopromoción de la propia perfección y el no mostrar las imperfecciones las facetas más asociadas con la ansiedad.

Más allá de este desacuerdo, que indica la necesidad de continuar investigando, en la investigación de Flett y colaboradores (2012) se ha visto que la autopresentación perfeccionista se asocia con la ansiedad de forma independiente al perfeccionismo. En este mismo estudio se hallaron algunas correlaciones diferenciales para las distintas facetas y los componentes de la ansiedad: la faceta de no mostrar las imperfecciones estuvo relacionada con la evitación social y el malestar ante las situaciones nuevas, mientras que la autopromoción de la propia perfección se relacionó con la evitación social generalizada. Según los autores ya citados, estos hallazgos pueden indicar que, aunque sea más activa, esta faceta también comprende una tendencia a rehuir de situaciones en las que se puedan manifestar equivocaciones.

Es posible que las relaciones sociales sean un ámbito en el que estos niños y adolescentes sientan la amenaza de tener desempeños poco exitosos, y previenen los fracasos evitando entrar en contacto con los demás. Goya Arce y Polo (2016) encontraron que la ansiedad social está asociada a la autopresentación perfeccionista, y concluyen que esta podría ser una manifestación de la sensibilidad interpersonal de estos niños y adolescentes, es decir, de aquella tendencia a interpretar negativamente las experiencias interpersonales que forma parte del modelo de desconexión social. Por lo tanto, estos mismos autores arguyen que, más allá del malestar que genera en sí misma, la ansiedad social aumenta la vulnerabilidad a experimentar problemas interpersonales (como la soledad) que a su vez llevan al desarrollo de otros problemas de salud psicológica.

\section{Autopresentación perfeccionista y trastornos de la conducta alimentaria}

El perfeccionismo se encuentra relacionado con el desarrollo y el mantenimiento de trastornos de la alimentación, especialmente la anorexia y la bulimia nerviosa (Slof-Op't Landt, Claes y Van Furth, 2016), siendo un importante predictor de factores como preocupación por la dieta y la musculación en varones y preocupación por la dieta, la musculación y la comida en mujeres ( $\mathrm{Sa}-$ ling, Ricciardelli y McCabe, 2005).

La revisión bibliográfica reveló que la autopresentación perfeccionista constituye un factor clave que interviene no sólo en el inicio sino también en el mantenimiento y recuperación de los trastornos de la conducta alimentaria. Esta aseveración es válida tanto para la anorexia, la bulimia y los atracones (Boone, 2013; Castro et al., 2004).

Durante la adolescencia, el perfeccionismo genera la necesidad de mostrarse de manera perfecta ante los demás (i.e., sin ningún defecto corporal), lo que lleva a la persona a la ejecución de prácticas poco saludables que terminan configurando el origen y desarrollo de patologías alimenticias (Castro et al., 2004). Así, por ejemplo, las adolescentes que promocionan su propia perfección y que no muestran sus errores pueden preocuparse de forma disfuncional por el cuidado del cuerpo con el objetivo de acomodarse a las presiones sociales percibidas del entorno, principalmente de sus pares (Nanu y Scheau, 2013).

La autopresentación perfeccionista define la expresión social del perfeccionismo (Ferreira, et al., 2015), actuando como un mecanismo compensatorio ante sentimientos de inferioridad, inadecuación o miedo al rechazo que pueden surgir a partir de un estilo de apego inseguro (Boone, 2013; Hewitt et. al., 2003). El control sobre la alimentación puede emerger entonces como una estrategia desadaptativa para lidiar con la vergüenza, cuando se cree que presentar una imagen corporal perfecta lleva a lograr la valoración y aceptación de otros (Ferreira et al., 2015). Las facetas de autopromoción perfeccionista y no mostrar imperfecciones están particularmente implicadas con los trastornos de la alimentación, tanto en pacientes parcialmente recuperados como en aquellos que se encuentran en la fase activa de la enfermedad (Bardone-Cone et al., 2010). Asimismo, la faceta de no revelación de la imperfección se presenta como un factor común a los diagnósticos de trastornos de la alimentación, tanto para los pacientes en fase activa de la enfermedad, como para los parcialmente recuperados y los recuperados en su totalidad (Bardone-Cone et al., 2010).

Cabe destacar que en el estudio de Gan, Mohamad y Law (2018) la autopresentación perfeccionista no fue un predictor de los atracones. Estos resultados son opuestos a los obtenidos en los demás artículos científicos revisados y pueden deberse a diferencias culturales ya que el estudio fue realizado con población de adolescentes malayos. Por lo tanto, reviste interés científico avanzar en estudios acerca del impacto que tienen las condiciones contextuales y culturales en relación a la autopresentación perfeccionista. 


\section{Autopresentación perfeccionista y depresión}

La revisión de la bibliografía científica indica que los niños y adolescentes que se autopresentan de manera perfeccionista suelen presentar síntomas de tipo internalizantes (Lyman y Luthar, 2014). Particularmente, las tres facetas la autopresentación perfeccionista resultaron correlacionadas significativamente con síntomas de depresión (Castro et al., 2004; Hewitt et al., 2011)

En consistencia con el modelo de desconexión social expandido (Sherry, Mackinnon y Gautreau, 2016), el camino desde la autopresentación perfeccionista hasta la depresión está mediado por desconexión social objetiva y subjetiva. Las evidencias indican que, como ya se dijo, los adolescentes que se autopresentan de manera perfeccionista tienden a experimentar sensibilidad interpersonal en la forma de ansiedad social, que aumenta sus sentimientos de soledad, y a su vez los predispone para el desarrollo de sentimientos depresivos (Goya Arce y Polo, 2016).

También se estudió la autopresentación perfeccionista en una muestra clínica de adolescentes y se encontró que la tendencia a recurrir a una autopresentación perfeccionista puede aumentar el riesgo de suicidio, también por intermedio de desconexión social objetiva, expresada en haber sido víctima de bullying de parte de los pares, y desconexión social subjetivamente experimentada como sentimientos de desesperanza social (Roxborough et al., 2012).

\section{Discusión}

En este trabajo se realizó una revisión de la literatura científica sobre la autopresentación perfeccionista en niños y adolescentes con el objetivo de seleccionar los principales estudios empíricos que permitan comprender las relaciones entre el perfeccionismo y la autopresentación perfeccionista, los correlatos psicológicos y sociales de esta última, y sus asociaciones con psicopatología.

En la revisión de la literatura científica se ha observado la escasez de estudios empíricos acerca de esta temática. Debido a las consecuencias negativas de la autopresentación perfeccionista en la vida de niños y adolescentes se evidencia la importancia de avanzar con las investigaciones sobre este constructo. Estas investigaciones podrán contribuir en multiplicidad de ámbitos como la clínica, la escuela y las familias donde los niños y adolescentes con este estilo interaccional sufren en silencio para no mostrar sus imperfecciones.

Tras el análisis de los estudios se puede describir a la autopresentación perfeccionista como una de las estrategias que los perfeccionistas desadaptativos emplean para sobrellevar las consecuencias sociales de su estilo de apego inseguro, entre las que se encuentran: la necesidad de ser aceptado, la idea de que la autovaloración es contingente a los logros, la escasa resistencia a la influencia de los pares, y los esquemas desadaptativos tempranos sobre las propias imperfecciones. De esta forma, para conseguir la aprobación de los demás estos autopresentadores se esfuerzan por aparentar perfección, ya sea promocionando una imagen ideal de sí mismos y/o ocultando los errores.

Si bien se relaciona con el perfeccionismo auto-orientado y el socialmente prescrito, la autopresentación perfeccionista tiene características propias que le dan entidad de un constructo diferenciado que merece ser estudiado y comprendido en su singularidad. Las diferencias individuales serán las que marquen la preferencia por una u otra de sus facetas, que darán como resultado la preeminencia de los esfuerzos por mostrar la perfección o por ocultar las imperfecciones.

El afán por parecer perfecto, que emana del deseo de ser aceptado, deteriora la vida interpersonal dando lugar a la experiencia de desconexión social objetiva y subjetiva. De esta forma, aumenta la vulnerabilidad de los niños y adolescentes a desarrollar problemas como la ansiedad social, los trastornos de la conducta alimentaria y la depresión.

Si bien las distintas facetas de la autopresentación perfeccionista muestran patrones diferenciales de relación con algunas variables como la desconexión social subjetiva y objetiva, la ansiedad, la experiencia de emociones positivas y la reactividad a las situaciones negativas, los estudios analizados no aportan datos concluyentes que permitan distinguir si alguna de estas facetas es más o menos saludable que las demás.

Sin embargo, las evidencias del aumento de la vulnerabilidad psicológica asociada a la autopresentación perfeccionista en niños y adolescentes, y sus asociaciones con diversos problemas psicopatológicos denotan no sólo la relevancia del estudio de la autopresentación perfeccionista en relación a trastornos mentales, sino también la necesidad de incluirla en un abordaje conjunto durante el tratamiento de los mismos.

La investigación sobre esta temática es muy reciente, pero se inscribe como una línea interesante para conocer más sobre los problemas psicológicos que obstaculizan el desarrollo saludable de niños y adolescentes. Al haber mostrado asociaciones con el bullying resulta de gran valor continuar investigando en esta línea para mejorar la experiencia de los niños y adolescentes en sus años escolares, donde, además, todos los jóvenes se ven en cierta medida expuestos a la tendencia de procurar aparecer perfectos ante la mirada de los demás debido a que se promueve un estilo enfocado en los logros. 
Aun así, el impacto que tiene la autopresentación perfeccionista sobre la vida interpersonal plantea diversos interrogantes como, por ejemplo, cuáles son sus asociaciones con el uso de las redes sociales, cómo son las habilidades sociales de estos autopresentadores y qué tipo de apoyo social perciben y reciben cuando sienten malestar, siendo que evitan mostrarlo para no manchar su imagen de perfección. Si bien se han encontrado indicios del origen de este estilo interpersonal en los estilos de apego, también puede resultar valioso conocer las prácticas de crianza que favorecen su uso.

Profundizar en el conocimiento de la autopresentación perfeccionista podría promover el desarrollo, la adaptación y la validación de instrumentos que permitan su detección temprana, así como el desarrollo de estrategias de prevención y de intervención adecuadas. Por lo tanto, el estudio de la autopresentación perfeccionista en población infantil y adolescente resulta un campo fecundo de estudio e investigación, con implicaciones no sólo para el ámbito científico sino también para la clínica y la escuela.

\section{Conflicto de intereses}

Las autoras declaran que no existen conflictos de intereses.

\section{Referencias}

Bardone-Cone, A.M., Sturm, K., Lawson, M.A., Robinson, D.P., \& Smith, R. (2010). Perfectionism across stages of recovery from eating disorders. International Journal of Eating Disorders, 43(2), 139-148.

Baumeister, R.F. \& Hutton, D.G. (1987). Self-presentation theory: Self-construction and audience pleasing. In B. Mullen \& G.R. Goethals (Eds.), Theories of group behavior (pp. 71-87). New York, EEUU: Springer.

Beck, A.T., Rush, J., Shaw, B., y Emery, G. (2008). Terapia cognitiva de la depresión (18 $8^{\circ}$ ed.). Bilbao, España: Desclée de Brower.

Boone, L. (2013). Are attachment styles differentially related to interpersonal perfectionism and binge eating symptoms? Personality and individual differences, 54(8), 931-935.

Caputto, I., Cordero, S., Keegan, E., y Arana, F. (2015). Perfeccionismo y esquemas desadaptativos tempranos: un estudio con estudiantes universitarios. Ciencias Psicológicas, $9(2), 245-257$

Castro, J., Gila, A., Gual, P., Lahortiga, F., Saura, B., \& Toro, J. (2004). Perfectionism dimensions in children and adolescents with anorexia nervosa. Journal of Adolescent Health, 35(5), 392-398.

Chen, C., Hewitt, P.L., \& Flett, G.L. (2015). Preoccupied attachment, need to belong, shame, and interpersonal perfectionism: An investigation of the Perfectionism Social Disconnection Model. Personality and Individual Differences, 76(2015), 177-182.
Chen, C., Hewitt, P.L., Flett, G.L., Cassels, T.G., Birch, S., \& Blasberg, J.S. (2012). Insecure attachment, perfectionistic self-presentation, and social disconnection in adolescents. Personality and Individual Differences, 52(8), 936-941.

D'Alessandro, D.U. \& Burton, K.D. (2006). Development and validation of the Dysfunctional Attitudes Scale for Children: Tests of Beck's cognitive diathesis-stress theory of depression, of its causal mediation component, and of developmental effects. Cognitive Therapy and Research, 30(3), 335-353.

Deci, E.L. \& Ryan, R.M. (2012). Motivation, personality, and development within embedded social contexts: An overview of self-determination theory. In R.M. Ryan (Ed.), Oxford handbook of human motivation (pp. 85-107). Oxford, UK: Oxford University Press.

Ferreira, C., Trindade, I.A., \& Ornelas, L. (2015). Exploring drive for thinness as a perfectionistic strategy to escape from shame experiences. The Spanish Journal of Psychology, 18(e29), 1-7.

Flett, G.L., Coulter, L.M., \& Hewitt, P.L. (2012). The Perfectionistic Self-Presentation Scale-Junior Form: Psychometric properties and association with social anxiety in early adolescents. Canadian Journal of School Psychology, 27(2), 136-149.

Flett, G.L., \& Hewitt, P.L. (2013). Disguised distress in children and adolescents «flying under the radar» why psychological problems are underestimated and how schools must respond. Canadian Journal of School Psychology, 28(1), 12-27.

Gan, W.Y., Mohamad, N., \& Law, L.S. (2018). Factors Associated with Binge Eating Behavior among Malaysian Adolescents. Nutrients, 10(1), 66.

Goya Arce, A.B. \& Polo, A.J. (2016). A Test of the Perfectionism Social Disconnection Model among Ethnic Minority Youth. Journal of Abnormal Child Psychology, 45(6), 1181-1193.

Hewitt, P.L., Blasberg, J.S., Flett, G.L., Besser, A., Sherry, S.B., Caelian, C., Papsdorf, M., Cassels, T.G., \& Birch, S. (2011). Perfectionistic self-presentation in children and adolescents: Development and validation of the Perfectionistic SelfPresentation Scale-Junior Form. Psychological Assessment, 23(1), 125-142.

Hewitt, P.L. \& Flett, G.L. (1991). Perfectionism in the self and social contexts: conceptualization, assessment, and association with psychopathology. Journal of Personality and Social Psychology, 60 (3), 456-470.

Hewitt, P.L., Flett, G.L., \& Ediger, E. (1995). Perfectionism traits and perfectionistic self-presentation in eating disorder attitudes, characteristics, and symptoms. International Journal of Eating Disorders, 18(4), 317-326.

Hewitt, P.L., Flett, G.L., Sherry, S.B., \& Caelian, C. (2006). Trait perfectionism dimensions and suicide behavior. In T.E. Ellis (Ed.), Cognition and Suicide. Theory, Research and Therapy (pp. 215-235). Washington DC, EEUU: American Psychological Association.

Hewitt, P.L., Flett, G.L., Sherry, S.B., Habke, M., Parkin, M., Lam, R.W., McMurtry, B., Ediger, E., Fairlie, P., \& Stein, M.B. (2003). The interpersonal expression of perfection: Perfectionistic selfpresentation and psychological distress. Journal of Personality and Social Psychology, 84 (2), 1303-1325.

James, K., Verplanken, B., \& Rimes, K.A. (2015). Self-criticism as a mediator in the relationship between unhealthy perfectionism and distress. Personality and Individual Differences, 79(2015), 123-128. 
Jahromi, F.G., Naziri, G., \& Barzegar, M. (2012). The relationship between socially prescribed perfectionism and depression: The mediating role of maladaptive cognitive schemas. Procedia-Social and Behavioral Sciences, 32(2012), 141-147.

Lyman, E.L., \& Luthar, S.S. (2014). Further evidence on the «costs of privilege»: Perfectionism in high-achieving youth at socioeconomic extremes. Psychology in the Schools, 51(9), 913-930.

Maloney, G.K., Egan, S.J., Kane, R.T., \& Rees, C.S. (2014). An etiological model of perfectionism. PloS one, 9(5), 1-20.

Nanu, E. \& Scheau, I. (2013). Perfectionism dimensions and resistance to peer influences in adolescence. Procedia-Social and Behavioral Sciences, 82(2013), 278-281.

Roxborough, H.M., Hewitt, P.L., Kaldas, J., Flett, G.L., Caelian, C.M., Sherry, S., \& Sherry, D.L. (2012). Perfectionistic selfpresentation, socially prescribed perfectionism, and suicide in youth: A test of the perfectionism social disconnection model. Suicide and Life-Threatening Behavior, 42(2), 217-233.

Saling, M., Ricciardelli, L.A. \& McCabe, M.P. (2005). A prospective study of individual factors in the development of weight and muscle concerns among preadolescent children. Journal of Youth and Adolescence, 34(6), 651-661.

Sherry, S.B., Hewitt, P.L., Flett, G.L., Lee-Baggley, D.L., \& Hall, P.A. (2007). Trait perfectionism and perfectionistic selfpresentation in personality pathology. Personality and Individual Differences, 42(3), 477-490.
Sherry, S.B., Mackinnon, S.P., \& Gautreau, C.M. (2016). Perfectionists don't play nicely with others: Expanding the social disconnection model. In F.M. Sirois \& D.S. Molnar (Eds.), Perfectionism, health, and well-being (pp. 225-243). New York, EEUU: Springer.

Slof-Op't Landt, M.C.T., Claes, L., \& Van Furth, E.F. (2016). Classifying eating disorders based on «healthy» and «unhealthy» perfectionism and impulsivity. International Journal of Eating Disorders, 49(7), 673-680.

Smith, R.H., \& Kim, S.H. (2007). Comprehending envy. Psychological Bulletin, 133(1), 46-64.

Stoeber, J., \& Roche, D.L. (2014). Affect intensity contributes to perfectionistic self-presentation in adolescents beyond perfectionism. Journal of Rational-Emotive y CognitiveBehavior Therapy, 32(2), 164-180.

Vuyk, M.A. (2015). Perfeccionismo, autopresentación perfeccionista, y síntomas depresivos en adolescentes Paraguayos según género y grado. Interamerican Journal of Psychology, 49(2), 153-163.

Wu, T.F. \& Wei, M. (2008). Perfectionism and negative mood: The mediating roles of validation from others versus self. Journal of Counseling Psychology, 55(2), 276.

Young, J.E., Klosko, J.S., \& Weishaar, M.E. (2003). Schema therapy: A practitioner's guide. New York, EEUU: Guilford Press. 
